\title{
Impact of Intraoperative Fluid Management on Electrolyte and Acid-Base Variables During Posterior Spinal Fusion in Adolescents
}

This article was published in the following Dove Press journal: Orthopedic Research and Reviews

\author{
Meagan King (D) $^{1,2}$ \\ David Martin ${ }^{1,3}$ \\ Renata Miketic ${ }^{1,3}$ \\ Allan Beebe ${ }^{4}$ \\ Walter Samora ${ }^{4}$ \\ Jan Klamar ${ }^{4}$ \\ Dmitry Tumin ${ }^{5}$ \\ Joseph D Tobias $\mathbb{D}^{1,3}$ \\ 'Department of Anesthesiology \& Pain \\ Medicine, Nationwide Children's \\ Hospital, Columbus, OH, USA; \\ ${ }^{2}$ Department of Anesthesiology, \\ Cincinnati Children's Hospital Medical \\ Center, Cincinnati, OH, USA; \\ ${ }^{3}$ Department of Anesthesiology \& Pain \\ Medicine, The Ohio State University \\ College of Medicine, Columbus, $\mathrm{OH}$, \\ USA; ${ }^{4}$ Department of Orthopedic \\ Surgery, Nationwide Children's Hospital \\ and the Ohio State University, Columbus, \\ $\mathrm{OH}$, USA; ${ }^{5}$ Department of Pediatrics, \\ Brody School of Medicine at East \\ Carolina University, Greenville, NC, USA
}

Correspondence: Joseph D Tobias Department of Anesthesiology \& Pain Medicine, Nationwide Children's Hospital, Columbus, OH, USA

Email Joseph.

Tobias@nationwidechildrens.org
Introduction: Various isotonic fluids may be used to maintain intravascular homeostasis during major surgical procedures. Variations in the electrolyte and buffer concentrations between these solutions may result in differential changes in electrolyte and acid-base status during fluid resuscitation. This study evaluates these changes during posterior spinal fusion in adolescents.

Methods: Patients were randomized to receive lactated Ringers (LR), normal saline (NS) or Normosol-R ${ }^{\circledR}(\mathrm{NR})$ during posterior spinal fusion $(\mathrm{N}=19,20$, and 20 , respectively). The specific fluid was used for maintenance fluids as well as fluid replacement of deficits, third space losses, and blood loss.

Results: Patients who received NS had a greater base deficit (NS: $-2.0 \pm 2.2$ vs NR $-0.6 \pm$ $1.8, \mathrm{p}=0.031$ or LR: $-0.2 \pm 1.7, \mathrm{p}=0.007$ ) and were more likely to have a $\geq 2$ point change in the base deficit ( $60 \%$ with NS compared to $30 \%$ with NR and $47 \%$ with LR). Patients receiving NS also had a lower $\mathrm{pH}(\mathrm{NS}: 7.37 \pm 0.03$ vs NR: $7.39 \pm 0.04, \mathrm{p}=0.013$ ) and a greater change in $\mathrm{pH}(\mathrm{NS}:-0.03 \pm 0.04$ vs NR: $0.01 \pm 0.06)$.

Conclusion: The use of NS for intraoperative resuscitation during posterior spinal fusion in adolescents resulted in a greater base deficit and a lower $\mathrm{pH}$ than the use of LR or NR. Although these changes had limited clinical significance in our patient population, future studies are indicated to further investigate the potential clinical impact of these changes.

Keywords: posterior spinal fusion, intravenous fluids, normal saline, dilutional acidosis

\section{Introduction}

During major surgical procedures, intravascular volume is maintained with the administration of isotonic fluids which may include lactated Ringers (LR), normal saline (NS) or Normosol-R® (NR). Although these fluids are all considered isotonic, they contain varying amounts of sodium and chloride, as well as having different buffers to maintain a neutral $\mathrm{pH}^{1,2}$ There is no buffer in NS; acetate and gluconate are present in NR, and lactate in LR. Given the absence of buffer in NS and the different buffers in the other two solutions, there may be variations in the acid-base and electrolyte changes following intraoperative resuscitation during

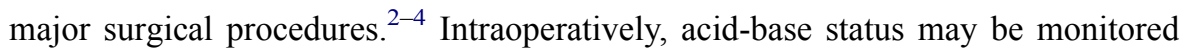
as an indirect measure of intravascular resuscitation and tissue perfusion. Inadequate resuscitation may result in an increasing lactic acid concentration and an increasing base excess. Increasing base deficits may also occur due to the administration of large volumes of NS, which has been termed dilutional acidosis. 
Dilutional acidosis occurs when the endogenous buffer system is diluted by non-buffer containing intravenous fluids, thus resulting in a hyperchloremic metabolic acidosis. The difference is not easily discernible on standard arterial blood gas (ABG) analysis and may require additional testing such as measurement of a serum lactate value.

As NS administration may result in hyperchloremic metabolic acidosis and an increasing base deficit, intraoperative clinical decisions may be challenging when guided solely by acid-base status on routine ABG analysis. Additionally, although the hyperchloremic metabolic acidosis has generally been considered benign, recent studies from critically ill adult patients in both the intensive care unit (ICU) and operating room suggest that this acidosis is independently associated with increased mortality in at-risk patients. ${ }^{5,6}$ The administration of NS and the resultant hyperchloremia may decrease renal blood flow and glomerular filtration rate, and may be associated with an increased incidence of acute kidney injury in critically ill adults. ${ }^{7,8}$ The current study evaluated changes in acid-base status and electrolytes (sodium, potassium, calcium) during posterior spinal fusion with intraoperative fluid resuscitation using three different isotonic fluids. We hypothesized that a fluid containing a buffer (LR or NR) would mitigate these changes during intraoperative care.

\section{Methods}

This prospective, randomized trial was approved by the Institutional Review Board of Nationwide Children's Hospital (IRB16-01093) in accordance with the Declaration of Helsinki. Deidentified data supporting this study will be archived and kept indefinitely, but will not be available unless required to support the findings of this study. Patients and parents were informed of the purpose of the study and written consent was obtained from a parent or guardian and assent from the patient. The study was registered at Clinicaltrials.gov (NCT03054922) and the study team enrolled patients over an 18-month period. After consent and enrollment, adolescent patients presenting for posterior spinal fusion surgery for the treatment of either neuromuscular or idiopathic scoliosis were randomized to receive either NS, LR, or NR during intraoperative care. During the study phase of the anesthetic care, the study fluid was used for all aspects of intraoperative fluid therapy including the provision of maintenance fluids as well as replacement of deficits, third space losses, and blood loss. Fluid was administered as needed by the anesthesia team based on clinical parameters including heart rate (HR), blood pressure, urine output, and estimated blood loss. ABGs, electrolytes, hemoglobin, and lactic acid were measured at the start of surgery and either at the end of the surgical procedure or when there was a clinical need for another type of fluid (colloid) or blood. At the same time that the ABG was obtained, the HR and mean arterial pressure (MAP) were recorded. ABG, hemoglobin, lactic acid, and electrolytes were analyzed using point-of-care monitoring in the operating room.

The anesthetic technique followed our department's standardized intraoperative anesthetic care for posterior spinal fusion. ${ }^{9}$ The technique, as previously described, has been developed to allow for effective monitoring of motor (MEP) and somatosensory evoked potentials and yet allow for a rapid wake-up test if needed. The patients were held nil per os for 6-8 hrs prior to surgery. Premedication included oral or intravenous midazolam followed by either inhalational or intravenous anesthetic induction. For inhalation induction, the patient breathed increasing increments of sevoflurane in nitrous oxide and oxygen until intravenous access was achieved. For intravenous induction, a peripheral intravenous cannula was placed after the patient breathed nitrous oxide for 2-3 mins. The administration of either sevoflurane or nitrous oxide lasted no more than 3 mins. Following this, maintenance anesthesia was the same for all patients and included desflurane (inspired concentration adjusted to maintain the bispectral index at 50-60) and sufentanil or remifentanil with methadone. Neuromuscular blockade was provided by intermittent dosing of rocuronium as allowed according to MEP monitoring. The sufentanil or remifentanil infusion was adjusted as needed up to $0.5 \mu \mathrm{g} /$ $\mathrm{kg} / \mathrm{min}$ for remifentanil or $0.5 \mu \mathrm{g} / \mathrm{kg} /$ hour for sufentanil to maintain the MAP at the desired level. Labetalol $(0.1-0.15 \mathrm{mg} / \mathrm{kg} /$ dose $)$ was administered as needed to control the MAP. Following anesthetic induction, an arterial cannula and two peripheral intravenous cannulas were placed. An ABG was obtained after anesthetic induction, prior to the start of the surgical procedure and the administration of fluid resuscitation. Additional ABGs were obtained as clinically indicated, and a final ABG was obtained and the study period concluded prior to the administration of blood, including cell saver, blood products, colloid (5\% albumin) or anything other than the study fluid.

The primary outcome was an increase in base deficit $\geq$ $2 \mathrm{mEq} / \mathrm{L}$ between NS and LR and between NS and NR. 
The incidence of base deficit in excess of $-2 \mathrm{mEq} / \mathrm{L}$ was compared between NS and LR and between NS and NR using tests of independent proportions. In a pilot study enrolling 48 patients, we found that the incidence of base deficit in excess of $-2 \mathrm{mEq} / \mathrm{L}$ was $15 \%$. This study was originally powered to detect a difference in the incidence of $25 \%$ ( $15 \%$ vs $40 \%$ ) between NS and either LR or NR, with the overall Type I error set at 0.05 and the significance level of individual tests (NS vs LR; NS vs NR) adjusted for multiple comparisons using the Bonferroni correction. To attain $80 \%$ power for detecting this expected difference, two-tailed tests of independent proportions required 60 patients in each group. The study was curtailed after 59 patients were enrolled, due to slow progress of enrollment.

Categorical data were analyzed using independent tests of proportions or Fisher's exact tests, and continuous data were analyzed using independent $t$-tests. Secondary variables measured included changes in electrolyte values, volume of intraoperative fluid requirements, intraoperative urine output, and intraoperative hemodynamic variables including MAP and HR. A Pearson correlation was used to examine the correlation between hemoglobin and base deficit and between fluid administration and change in base deficit, according to fluid type. Analysis of variance (ANOVA) was used to compare MAP and HR between the two groups. When comparing base deficit changes, a twotailed $\mathrm{p}<0.025$ was considered statistically significant to account for multiple comparisons (NS vs LR and NS vs NR).

\section{Results}

The study cohort included 59 patients (40 female, 19 male), who were randomized 20 to NR, 20 to NS, and 19 to LR. For the study cohort, the mean age was $14 \pm 2$ years and the mean weight was $58 \pm 17 \mathrm{~kg}$ (Table 1). There were no differences in age, weight, body mass index, gender or American Society of Anesthesiologists (ASA) physical status between the 3 groups, except that there was an over-representation of males in the LR group (53\% in LR versus $85 \%$ in NS). Base deficit was measured at the beginning of the case and either at the end of the surgical procedure or when there was a clinical need for another type of fluid (colloid). Of the 59 patients, $\mathrm{BD}$ became more negative in 38 patients during the procedure. Patients who received NS had a statistically significant increase in $\mathrm{BD}$ when compared to those who received LR or NR. Furthermore, 27 patients had an increase in $\mathrm{BD} \geq 2,12$ of whom received NS compared to 6 who received NR and 9
Table I Demographics of the Study Cohort $(\mathrm{N}=59)$

\begin{tabular}{|c|c|c|c|}
\hline \multirow[t]{2}{*}{ Characteristics } & \multicolumn{3}{|c|}{ Fluid Type } \\
\hline & $\begin{array}{l}N R \\
(N=20)\end{array}$ & $\begin{array}{l}\text { NS } \\
(N=20)\end{array}$ & $\begin{array}{l}\text { LR } \\
(\mathrm{N}=19)\end{array}$ \\
\hline Female & 14 (70\%) & 17 (85\%) & $10(53 \%)$ \\
\hline $\begin{array}{l}\text { Neuromuscular/idiopathic } \\
\text { scoliosis }\end{array}$ & $4 / 16$ & $2 / 18$ & $8 / 11$ \\
\hline Age (years) & $14 \pm 2$ & $14 \pm 3$ & $14 \pm 2$ \\
\hline Height $(\mathrm{cm})$ & $162 \pm 11$ & $162 \pm 9$ & $157 \pm 10$ \\
\hline Weight (kg) & $57 \pm 18$ & $61 \pm 13$ & $57 \pm 19$ \\
\hline Body mass index $\left(\mathrm{kg} / \mathrm{m}^{2}\right)$ & $22 \pm 6$ & $23 \pm 5$ & $22 \pm 6$ \\
\hline \multicolumn{4}{|l|}{ ASA status } \\
\hline 1 & $5(25 \%)$ & 7 (35\%) & $2(11 \%)$ \\
\hline 2 & II (55\%) & $10(50 \%)$ & II (6I\%) \\
\hline 3 & $4(20 \%)$ & $3(15 \%)$ & $5(28 \%)$ \\
\hline $\begin{array}{l}\text { Surgery or study time } \\
\text { (minutes) }\end{array}$ & $271 \pm 83$ & $259 \pm 72$ & $247 \pm 94$ \\
\hline Estimated blood loss (mL/kg) & $\begin{array}{l}12.3 \pm \\
15.7\end{array}$ & $10.2 \pm 8.4$ & $7.5 \pm 4.9$ \\
\hline
\end{tabular}

Notes: The data are listed as the mean \pm standard deviation or the number (\%). There were no statistical differences among the three groups.

Abbreviations: ASA, American Society of Anesthesiologists; LR, lactated Ringers; NR, Normosol-R®; NS, normal saline.

who received LR. The difference in proportions between NS and NR did not reach the prespecified statistical significance threshold of $\mathrm{p}=0.025$ (difference $=30 \% ; 95 \%$ confidence interval $[\mathrm{CI}]$ of difference: $0 \%, 59 \%$; $\mathrm{p}=0.057$ ). Likewise, the difference in proportions between NS and LR did not reach statistical significance (difference $=17 \% ; 95 \%$ CI of difference: $-13 \%, 47 \%$; $\mathrm{p}=0.275$ ).

Compared to patients receiving either NR or LR, patients receiving NS had a greater $\mathrm{BD}$ at the second measurement (NS: $-2.0 \pm 2.2$ vs NR $-0.6 \pm 1.8, \mathrm{p}=0.031$ or LR: $-0.2 \pm 1.7$, $\mathrm{p}=0.007$ ) (Table 2). Compared to patients receiving NR, patients receiving NS had a lower $\mathrm{pH}$ at the second measurement (NS: $7.37 \pm 0.03$ vs NR: $7.39 \pm 0.04, p=0.013$ ) and a greater change in $\mathrm{pH}$ (NS: $-0.03 \pm 0.04$ vs NR: $0.01 \pm 0.06$ ) (Table 2). Differences in electrolytes, HR, MAP, total fluid administration, and total urine output were not statistically significantly different between the three groups except for a modest difference in the potassium change when comparing NR versus $L R(0.2 \pm 0.4$ versus $0.7 \pm 0.6 \mathrm{mEq} / \mathrm{L}$, $\mathrm{p}=0.007$ ) (Table 3 ).

\section{Discussion}

The current study demonstrates a statistically significant difference in the changes in acid-base status when comparing intraoperative fluid resuscitation with 3 commonly used 
Table 2 Base Deficit, $\mathrm{pH}$, and Electrolyte Changes in the Study Cohorts

\begin{tabular}{|c|c|c|c|c|c|c|}
\hline \multirow[t]{2}{*}{ Measurement } & \multicolumn{3}{|l|}{ Fluid Type } & \multicolumn{3}{|l|}{ P-value } \\
\hline & NR $(\mathbf{N}=20)$ & NS $(\mathbf{N}=20)$ & LR $(\mathbf{N}=19)$ & NR vs NS & NR vs LR & NS vs LR \\
\hline \multicolumn{7}{|c|}{ Base Deficit (mEq/L) } \\
\hline First $A B G$ & $-0.1 \pm 2.2$ & $-0.5 \pm 2.5$ & $0.9 \pm 2.5$ & 0.549 & 0.191 & 0.081 \\
\hline Second $A B G$ & $-0.6 \pm 1.8$ & $-2.0 \pm 2.2$ & $-0.2 \pm 1.7$ & $0.03 I$ & 0.550 & 0.007 \\
\hline Change & $-0.5 \pm 1.5$ & $-1.5 \pm 2.4$ & $-1.2 \pm 2.3$ & 0.120 & 0.301 & 0.650 \\
\hline \multicolumn{7}{|l|}{$\mathrm{pH}$} \\
\hline First $A B G$ & $7.39 \pm 0.05$ & $7.40 \pm 0.05$ & $7.40 \pm 0.05$ & 0.455 & 0.347 & 0.813 \\
\hline Second $A B G$ & $7.39 \pm 0.04$ & $7.37 \pm 0.03$ & $7.39 \pm 0.04$ & 0.013 & 0.435 & 0.094 \\
\hline Change & $0.01 \pm 0.06$ & $-0.03 \pm 0.04$ & $-0.02 \pm 0.06$ & 0.017 & 0.194 & 0.426 \\
\hline \multicolumn{7}{|c|}{ Electrolyte change (mEq/L) } \\
\hline $\mathrm{Na}^{+}$ & $-0.1 \pm 1.9$ & $0.1 \pm 1.7$ & $-0.4 \pm 1.5$ & 0.794 & 0.633 & 0.426 \\
\hline $\mathrm{K}^{+}$ & $0.2 \pm 0.4$ & $0.4 \pm 0.4$ & $0.7 \pm 0.6$ & 0.175 & 0.007 & 0.085 \\
\hline $\mathrm{Ca}^{++}$ & $-0.01 \pm 0.08$ & $0.01 \pm 0.05$ & $-0.13 \pm 0.57$ & 0.144 & 0.407 & 0.288 \\
\hline
\end{tabular}

Notes: The data are listed as the mean \pm SD. Analysis with a $P$ value less than 0.05 are listed in bold and italics.

Abbreviations: $\mathrm{ABG}$, arterial blood gas; $\mathrm{Na}^{+}$, sodium; $\mathrm{K}^{+}$, potassium; $\mathrm{Ca}^{++}$, calcium; $\mathrm{LR}$, lactated Ringers; $\mathrm{NR}, \mathrm{Normosol}-\mathrm{R} \circledast$; NS, normal saline.

Table 3 Hemodynamic, Fluid, Urine Output, and Lactate Variables Between the Study Groups

\begin{tabular}{|l|l|l|l|l|l|l|}
\hline \multirow{2}{*}{ Measurement } & \multicolumn{2}{l|}{ Fluid Type } & \multicolumn{2}{l|}{ P-value } \\
\cline { 2 - 7 } & NR (N=20) & NS (N=20) & LR (N=19) & NR vs NS & NR vs LR & NS vs LR \\
\hline HR change (beats/minute) & $0 \pm 13$ & $0 \pm 14$ & $5 \pm 22$ & 0.972 & 0.417 & 0.418 \\
MAP change (mmHg) & $-4 \pm 12$ & $0 \pm 13$ & $-2 \pm 14$ & 0.356 & 0.664 & 0.663 \\
Total fluid administration (mL/kg) & $35 \pm 23$ & $30 \pm 15$ & $48 \pm 87$ & 0.389 & 0.526 & 0.364 \\
Total urine output (mL/kg) & $6 \pm 4$ & $5 \pm 3$ & $5 \pm 4$ & 0.627 & 0.487 & 0.774 \\
\hline Lactate & & & & & \\
First ABG & $1.1 \pm 0.4$ & $1.2 \pm 0.7$ & $1.5 \pm 0.6$ & 0.428 & $\mathbf{0 . 0 2 I}$ & 0.250 \\
Final ABG & $1.2 \pm 0.5$ & $1.3 \pm 0.7$ & $1.5 \pm 0.7$ & 0.485 & 0.061 & 0.321 \\
Change & $0.1 \pm 0.4$ & $0.1 \pm 0.4$ & $0.1 \pm 0.6$ & 0.986 & 0.995 & 0.995 \\
\hline
\end{tabular}

Notes: The data are presented as the mean \pm SD. Analysis with a $p$ value less than 0.05 are listed in bold and italics.

Abbreviations: ABG, arterial blood gas; HR, heart rate; MAP, mean arterial pressure; LR, lactated Ringers; NR, Normosol-R®; NS, normal saline.

isotonic fluids during posterior spinal fusion in adolescents. The use of NS for intraoperative resuscitation resulted in a greater base deficit and a lower $\mathrm{pH}$ than the use of LR or NR. While all 3 of these fluids may be commonly used during major surgical procedures, the choice of fluid is likely based more on the discretion of the anesthesia team rather than evidence-based medicine. LR seems to be the time-honored fluid for intraoperative resuscitation in the absence of concerns of compromised intracranial compliance, when the safety of the lower sodium concentration has been questioned given its impact on serum osmolarity. ${ }^{10-12}$ While the impact of these changes on the eventual clinical outcome is likely limited in this relatively healthy adolescent population, it demonstrates that BD cannot be used as a surrogate indicator for volume resuscitation or changes in lactic acid related to end organ perfusion. Despite equal resuscitation volumes, resuscitation with NS resulted in an elevated $\mathrm{BD}$ and an iatrogenic dilutional acidosis when compared to other fluids. Given the variation in the length and severity of the surgical procedure, there were differences in the volume of isotonic fluid administered. However, despite the larger volumes used in the LR group when compared to the other two groups, these differences did not meet statistical significance. As the only significant clinical was noted in patients who received NS, we would postulate that the differences in the volume of isotonic fluids used did not impact the finding of a greater incidence of dilutional acidosis with NS.

In the adult population, there is concern that hyperchloremia may impact renal blood flow, glomerular filtration rate, 
resulting in an increased incidence of acute kidney injury. In the ICU population, reduction of chloride load resulted in a significant decrease in metabolic acidosis and normalization of acid-base status. ${ }^{4}$ Similar to findings in adult critical care populations, where metabolic acidosis has been shown to be an independent predictor of outcomes, this acidosis may lead to further iatrogenic harm and increased morbidity. This includes a significantly higher risk of acute kidney injury via afferent arteriolar vasoconstriction and reduced GFR, and longer mechanical ventilation time, which are associated with poor outcomes. ${ }^{5,8}$ In a meta-analysis of perioperative care in adults, hyperchloremia from high versus low-chloride-containing solutions also has been associated with impaired immune function, decreased renal perfusion, and increased mortality. An additional factor that may compromise postoperative outcomes is that the hyperchloremic metabolic acidosis from NS administration may further augment the decrease in $\mathrm{pH}$ from respiratory acidosis due to perioperative opioid use. The additive effects of the metabolic and respiratory acidosis may result in a $\mathrm{pH}$ less than $7.2 .^{2}$ As noted previously, clinical trials have demonstrated that due to the increase in BD and decrease in $\mathrm{pH}$ seen after bolus administration of hyperchloremic fluids, providers may be incorrectly treating the iatrogenic acidosis with additional amounts of crystalloid, believing it to be the result of hypovolemia and hypoperfusion, thereby further compromising outcomes.

In summary, the intraoperative administration of $\mathrm{pH}$ balanced intravenous fluids with serum-comparable amounts of chloride and buffers such as acetate or lactate resulted in a lower $\mathrm{BD}$, higher $\mathrm{pH}$, and minimal electrolyte changes when compared to NS for fluid resuscitation in adolescents undergoing posterior spinal fusion to treat scoliosis. Although the study was curtailed due to slow enrollment over a period of approximately 2 years and did not reach the planned sample size for a power analysis based on the categorical outcome, secondary analyses of continuous endpoints demonstrated consistently higher BD in the NS group. A multi-center prospective collaborative study may be needed to definitively confirm the association between fluid type and BD. Additionally, the clinical impact of these changes did not appear significant in our study. Future studies are indicated to further investigate the potential clinical impact of these changes and whether the hyperchloremia impacts end-organ function as has been demonstrated in the adult, critically ill patient. In clinical practice, the increasing BD may be viewed incorrectly as a sign of hypovolemia and the need for additional fluid resuscitation. Based on evidence from adult populations, physiologic principles of fluid resuscitation, and data from this study, dilutional acidosis can be largely prevented by the use of a balanced salt solution for routine fluid resuscitation.

\section{Disclosure}

Dr Walter Samora reports personal fees from Globus Medical and Depuy/Synthes, outside the submitted work. The authors report no other conflicts of interest in this work.

\section{References}

1. Awad S, Allison SP, Lobo DN. The history of $0.9 \%$ saline. Clin Nutr. 2008;27:179-188. doi:10.1016/j.clnu.2008.01.008

2. Scheingraber S, Rehm M, Sehmisch C, et al. Rapid saline infusion produces hyperchloremic acidosis in patients undergoing gynecologic surgery. Anesthesiology. 1999;90:1265-1270. doi:10.1097/00000542199905000-00007

3. Williams EL, Hildebrand KL, McCormick SA, et al. The effect of intravenous lactated Ringer's solution versus $0.9 \%$ sodium chloride solution on serum osmolality in human volunteers. Anesth Analg. 1999;88:999-1003. doi:10.1097/00000539-199905000-00006

4. Yunos NM, Kim IB, Bellomo R, et al. The biochemical effects of restricting chloride-rich fluids in intensive care. Crit Care Med. 2011;39:2419-2424. doi:10.1097/CCM.0b013e31822571e5

5. Krajewski ML, Raghunathan K, Paluszkiewicz SM, et al. Metaanalysis of high- versus low-chloride content in perioperative and critical care fluid resuscitation. $B r \quad J$ Surg. 2015;102:24-36. doi:10.1002/bjs.9651

6. Rocktaeschel J, Morimatsu H, Uchino S, et al. Unmeasured anions in critically ill patients: can they predict mortality? Crit Care Med. 2003;31:2131-2136. doi:10.1097/01.CCM.0000079819.27515.8E

7. Neyra JA, Canepa-Escaro F, Li X, et al. Acute kidney injury in critical illness study group: association of hyperchloremia with hospital mortality in critically ill septic patients. Crit Care Med. 2015;43:1938-1944. doi:10.1097/CCM.0000000000001161

8. Chowdhury AH, Cox EF, Francis ST, et al. A randomized, controlled, double-blind crossover study on the effects of 2-L infusions of $0.9 \%$ saline and Plasmalyte ${ }^{\circledR} 148$ on renal blood flow velocity and renal cortical tissue perfusion in healthy volunteers. Ann Surg. 2012;256:18-24. doi:10.1097/SLA.0b013e318256be72

9. Martin DP, Bhalla T, Thung A, et al. A preliminary study of volatile agents or total intravenous anesthesia for neurophysiological monitoring during posterior spinal fusion in adolescents with idiopathic scoliosis. Spine. 2014;39:E1318-24. doi:10.1097/BRS.00000000 00000550

10. Ramming S, Shackford SR, Zhuang J, Schmoker JD. The relationship of fluid balance and sodium administration to cerebral edema formation and intracranial pressure in a porcine model of brain injury. $J$ Trauma. 1994;37:705-713. doi:10.1097/00005373-19941100000003

11. Shackford SR, Zhuang J, Schmoker J. Intravenous fluid tonicity: effect on intracranial pressure, cerebral blood flow, and cerebral oxygen delivery in focal brain injury. J Neurosurg. 1992;76:91-98. doi:10.3171/jns.1992.76.1.0091

12. Sharma D, Vavilala MS. Perioperative management of adult traumatic brain injury. Anesthesiol Clin. 2012;30:333-346. doi:10.1016/j. anclin.2012.04.003 


\section{Publish your work in this journal}

Orthopedic Research and Reviews is an international, peer-reviewed, open access journal that focusing on the patho-physiology of the musculoskeletal system, trauma, surgery and other corrective interventions to restore mobility and function. Advances in new technologies, materials, techniques and pharmacological agents are particularly

Submit your manuscript here: https://www.dovepress.com/orthopedic-research-and-reviews-journa welcome. The manuscript management system is completely online and includes a very quick and fair peer-review system, which is all easy to use. Visit http://www.dovepress.com/testimonials.php to read real quotes from published authors. 